\title{
Modificações no Índice de Líquido Amniótico Estimado pela Ultra-sonografia em Gestantes Submetidas a Imersão Subtotal em Água
}

\author{
Changes in Amniotic Fluid Index Estimated by Ultrasonography in Pregnant Patients \\ Submitted to Subtotal Immersion in Water \\ Celso Engelberto Ayres, Francisco Mauad-FilhoAdilson Cunha Ferreira \\ Uilho Antonio Gomes, Luciano S. Pinheiro-Filho
}

\begin{abstract}
RESUMO
Objetivo: estudar as modificações do índice de líquido amniótico (ILA), mensuradas pela ultra-sonografia, da pressão arterial média (PAM) e do pulso em gestantes normais quando submetidas a imersão corporal subtotal em água, por diferentes intervalos de tempo, $e$ padronização da técnica.

Métodos: foram estudados os valores do ILA, como descrito por Phelan et al. ${ }^{1}$, de um grupo de 52 gestantes, com idade gestacional igual ou superior a 28 semanas, consideradas clinicamente normais, antes e após a sua imersão subtotal em um tanque com água aquecida entre $32^{\circ} \mathrm{C}$ e $34^{\circ} \mathrm{C}$, por 30,45 e 60 minutos.

Resultados: as médias da PAM, antes e depois da imersão, mostraram diferenças estatisticamente significantes: 87,8 e 87,1 no grupo de 30 minutos, 76, 7 e 66,6 no grupo de 45 minutos e 77,4 e 60,7 no grupo de 60 minutos. As médias de pulso também foram diferentes, com valores 74,9 e 78,7 no grupo de 30 minutos, 83,6 e 85,2 no grupo de 45 minutos e 84, 9 e 90,6 no grupo de 60 minutos. Quanto ao grupo que foi submetido a imersão por 30 minutos, o valor médio do ILA foi de $11,7 \mathrm{~cm}$ antes e de 16,8 cm após imersão. No grupo de 45 minutos, o valor médio do ILA foi de 9,7 cm antes e de 13,8 cm após a imersão. No grupo de 60 minutos, o valor médio do ILA foi de 9,5 cm antes e de 13,6 cm após a imersão. O tempo de imersão de 30 minutos é tão eficaz e suficiente quanto 45 e 60 minutos. Conclusões: a imersão subtotal de gestantes em água é segura, prática e capaz de imobilizar os fluidos durante a gestação, aumentando o volume de líquido amniótico.
\end{abstract}

PALAVRAS-CHAVE: Índice de líquido amniótico. Líquido amniótico. Gravidez normal.

Introdução

O ser humano está envolvido desde a sua formação pelo líquido amniótico (LA). A sua origem e o seu volume variam conforme a idade gestacional e dependem de uma série de trocas envolvendo o feto, a placenta, as membranas e o organismo materno. Apesar destas alterações serem nítidas, até o presente pouco se sabe sobre os mecanismos fisiológicos que regulam a sua composição e o seu volume nos diferentes trimestres gestacionais ${ }^{2}$.

Departamento de Ginecologia e Obstetrícia da Faculdade de Medicina de Ribeirão Preto - USP

Correspondência:

Francisco Mauad Filho

Av. Bandeirantes, $3900-8^{\circ}$ andar

14049-900 - Ribeirão Preto - SP
A deglutição é a principal via pela qual o fluido deixa a cavidade amniótica, sendo a micção fetal a maior fonte de LA, desde o início da segunda metade da gestação. Também é consenso que a manutenção de um volume variável de LA, durante toda a gestação, é importante para o bemestar fetal e um adequado indicador de normalidade gestacional ${ }^{3}$.

Até a década de 60, a estimativa do volume de LA, no periodo anteparto, era efetuada pela avaliação obstétrica ou com a realização da amniocentese e utilização de corantes ${ }^{4}$. Com o advento da ultra-sonografia tornou-se possivel a estimativa do volume de LA, bem como suas variações nos diferentes periodos gestacionais ${ }^{5}$ além de seu estudo semiquantitativo ${ }^{1}$.

Por outro lado, as anormalidades no volume 
de líquido amniótico (VLA) estão associadas com resultados gestacionais adversos. A oligoidramnia está associada a retardo de crescimento intrauterino, sofrimento fetal, gestação serotina e malformação letal, ao passo que a polidramnia se associa com malformações letais, isoimunização, diabete e gêmeos idênticos.

Vários estudos mostram que a expansão adequada da volemia materna apresenta correlação positiva com um volume normal de LA e que a oligoidramnia poderia resultar de uma expansão plasmática materna inadequada. Objetivando reverter esta complicação, tem-se preconizado a utilização de diferentes técnicas com o intuito de se aumentar o volume de líquido amniótico, na vigência de oligoidramnia. As mais conhecidas são o repouso, a hiper-hidratação materna por via oral ou endovenosa, a amnioinfusão e a imersão corporal subtotal em água aquecida ${ }^{6-10}$.

O objetivo do presente trabalho foi estudar as modificações do índice de líquido amniótico (ILA) com a ultra-sonografia, em gestantes de diferentes idades gestacionais, clinicamente normais, quando submetidas a imersão subtotal em água aquecida, por diferentes intervalos de tempo, visando a padronização desta técnica.

\section{Pacientes e Métodos}

Realizou-se estudo prospectivo com 52 gestantes atendidas na Escola de Ultra-sonografia e Reciclagem Médica Ribeirão Preto, em colaboração com a Faculdade de Medicina de Ribeirão Preto (FMRP-USP), no periodo de abril de 1995 a junho de 1997, tendo sido aprovado pelo Conselho de Ética do Hospital das Clínicas de Ribeirão Preto. Para este estudo foram utilizados como critérios de inclusão a presença de gestação única, idade gestacional acima de 24 semanas (definida pela data da última menstruação e pelos exames ultrasonográficos prévios) presença de bolsa amniótica integra e, como critérios de exclusão, a presença de cardiopatia e lesões de pele em atividade. Não se limitou a ingestão de água ou de alimentos, porém solicitava-se o esvaziamento vesical, antes e após a imersão em água aquecida, de todas as pacientes. As 52 participantes foram distribuídas em um grupo de 21 gestantes imersas por 30 minutos, outro grupo de 11 por 45 e o terceiro grupo com 20 gestantes teve 60 minutos de exposição.

A pressão arterial e o pulso, de cada uma das examinadas, foram aferidos pelo mesmo examinador, na posição sentada, dentro da água, durante e depois do banho. O banho de imersão foi realizado sempre em um mesmo tanque de fibra de vidro, com as medidas de $90 \times 70 \times 60 \mathrm{~cm}$ e com volume total de 250 litros, preenchido e mantido com água aquecida a $34^{\circ} \mathrm{C}$, monitorado por um termostato. A gestante era posicionada sentada, dentro do tanque com as pemas semi-fletidas, com água obrigatoriamente até a altura dos ombros, por períodos variáveis de tempo de 30,45 e 60 minutos.

O ILA foi mensurado pela ultra-sonografia, conforme técnica descrita por Phelan et al. ${ }^{1}$. Ela consiste em dividir em quatro quadrantes o abdomen gravídico materno através de linhas imaginárias longitudinal e transversa. A primeira utiliza a linha nigrans como eixo longitudinal e a segunda, a cicatriz umbilical como eixo transverso. Realiza-se a mensuração do diâmetro vertical do maior bolsão de cada quadrante e submete-se cada valor ao somatório, resultando no índice de líquido amniótico, ou ILA. Este valor é comparado a uma tabela, podendo ser qualificado como normal se entre 8,0 e 18,0 , oligoidramnia, se inferior a 5,0, intermediário, entre 5,0 e 8,0, aumentado, entre 18,0 e 24,0, e polidramnia, se superior a 24,0. Faz-se mister, durante a avaliação ultrasonográfica do líquido, não esquecer a morfologia fetal e uterina, que pode fornecer hipóteses para possiveis anormalidades encontradas.

Todos os exames foram realizados pelo mesmo observador. As participantes foram previamente orientadas sobre o protocolo da pesquisa e deram o seu consentimento.

\section{Resultados}

Todas as pacientes suportaram bem a imersão subtotal em água, na temperatura e em cada periodo de tempo estabelecidos acima. As médias da pressão arterial média (PAM), antes e depois da imersão, mostraram diferenças estatisticamente significantes: 87,8 e 87,1 no grupo de 30 minutos, 76,7 e 66,6 no grupo de 45 minutos e 77,4 e 60,7 no grupo de 60 minutos. As diferenças das médias de pulso também foram significantes, com valores 74,9 e 78,7 no grupo de 30 minutos, 83,6 e 85,2 no grupo de 45 minutos e 84,9 e 90,6 no grupo de 60 minutos ( $p=0,0014$ ) (Figuras 1 e 2).

Quando no estudo do grupo que foi submetido a imersão por 30 minutos, o valor médio do ILA foi de $11,7 \mathrm{~cm}$ antes e de $16,8 \mathrm{~cm}$ após o experimento. Os valores do ILA variaram de $3,0 \mathrm{~cm}$ a $22,5 \mathrm{~cm}$ antes e de $11,5 \mathrm{~cm}$ a $28,5 \mathrm{~cm}$ após o experimento. A média de variação dos valores do ILA foi de $5,1 \mathrm{~cm}$, com os extremos de $2,1 \mathrm{~cm}$ e 11,4 $\mathrm{cm}$. A porcentagem média de variação foi de $59,5 \%$. 


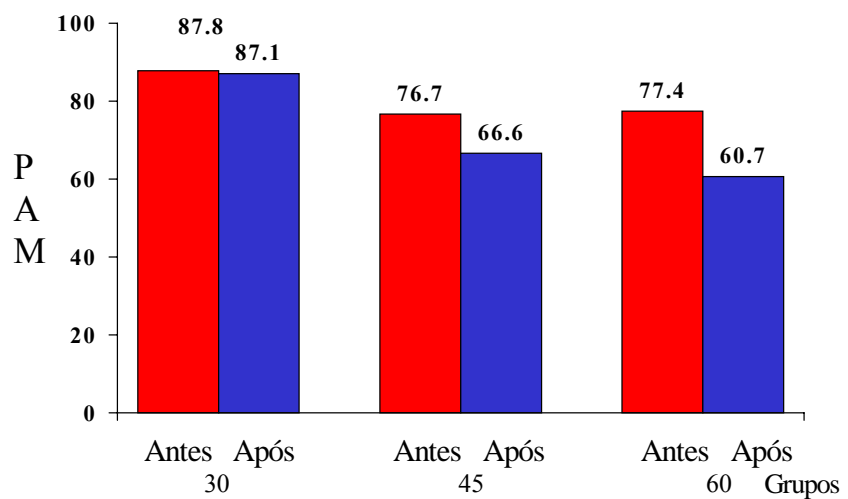

Figura 1 - Distribuição das médias da pressão arterial média (PAM) das gestantes antes e após a imersão subtotal em água aquecida, nos intervalos de tempo de 30, 45 e 60 minutos.

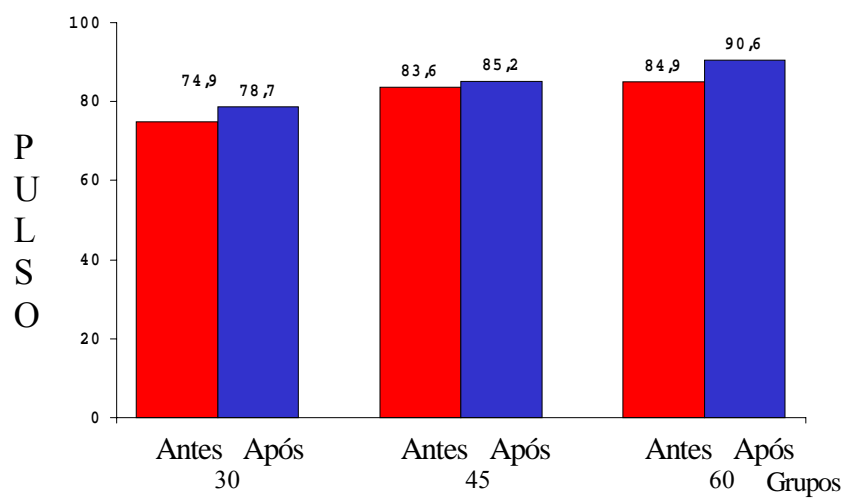

Figura 2 - Distribuição das médias do pulso arterial das gestantes antes e após a imersão subtotal em água aquecida, nos intervalos de tempo de 30, 45 e 60 minutos.

No grupo de 45 minutos, o valor médio do ILA foi de $9,7 \mathrm{~cm}$ antes e de $13,8 \mathrm{~cm}$ após o experimento. Os valores do ILA variaram de $3,0 \mathrm{~cm}$ a $22,5 \mathrm{~cm}$ antes e de $5,4 \mathrm{~cm}$ a $28,5 \mathrm{~cm}$ após o experimento. A média de variação dos valores do ILA foi de 4,0 cm, com os extremos de 2,2 cm e 9,2 cm. A porcentagem média de variação foi de $59,5 \%$.

No grupo de 60 minutos, o valor médio do ILA foi de $9,5 \mathrm{~cm}$ antes e de $13,6 \mathrm{~cm}$ após o experimento. Os valores do ILA variaram de $2,0 \mathrm{~cm}$ a $22,4 \mathrm{~cm}$ antes e de 4,2 $\mathrm{cm}$ a $25,6 \mathrm{~cm}$ após o experimento. A média de variação dos valores do ILA foi de 4,0 cm, com os extremos de 0,4 cm e 9,0 cm. A porcentagem média de variação foi de $49,1 \%$.

Aplicado o teste do $t$ pareado ao estudo da variação do ILA, mostrou-se que as médias do ILA apresentaram diferenças estatisticamente significantes no periodo de 30 minutos, $(p=0,001)$, no período de 45 minutos $(\mathrm{p}=0,001)$ e no período de 60 minutos $(p=0,0006)$ em relação à medida inicial (Figura 3).

É de relevância clinica enfatizar que todas as gestantes participantes referiram uma agradável sensação de bem-estar e uma acentuada melhora das dores nas costas e no baixo ventre, quando presentes, assim como uma percepção subjetiva de aumento dos movimentos fetais durante e após o banho, semelhante ao observado em outros relatos ${ }^{18}$.

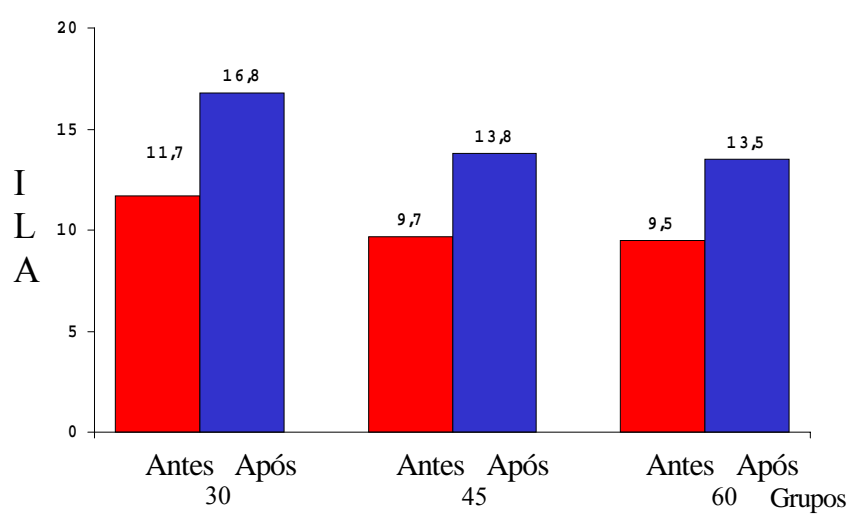

Figura 3 - Distribuição da média das variações do índice de líquido amniótico (ILA) das gestantes antes e após a imersão subtotal em água, por 30, 45 e 60 minutos.

\section{Discussão}

A composição e o VLA alteram-se conforme o evoluir da gravidez, mantendo o equilíbrio dinâmico do compartimento do saco amniótico. A imersão em água tem sido objeto de pesquisas em humanos na investigação dos efeitos fisiológicos da expansão do volume central sobre a função renal e as subseqüentes respostas hormonais ${ }^{1,7,11}$.

Acredita-se que o repouso no leito e/ou a imersão parcial do corpo humano em água leva a uma mobilização do fluido extravascular para o espaço intravascular, com redução do edema periférico, aumento da diurese, da natriurese e da caliurese, diminuição do peso corporal, diminuição da PAM e aumento no volume plasmático, na pressão venosa central e no débito cardíaco ${ }^{12-17}$. Neste estudo, a imersão em água produziu uma queda da PAM na maioria das pacientes que foram qualitativamente similares, mas quantitativamente diferentes nos três grupos examinados ${ }^{7-}$ ${ }^{12,18}$. O decréscimo da PAM é atribuído, por alguns autores, à mobilização do fluido extravascular para o intravascular e é caracterizado por um aumento da diurese das pacientes após o banho ${ }^{7,13,15}$.

A freqüência cardíaca média das gestantes aumentou em todos os grupos, diferente do encontrado em outros estudos que observaram ou ausência de variação ${ }^{18}$ ou um decréscimo significativo no pulso médio ${ }^{7,17}$. A explicação para o fato talvez esteja na metodologia ou na temperatura da água utilizada ${ }^{7,17,18}$.

Neste trabalho, encontramos alterações significativas no ILA em todos os grupos estudados. A 
média de variação esteve acima de $45 \%$ em todos os grupos, com variações individuais surpreendentes, acima de $300 \%$. Mesmo aquelas gestantes que apresentavam um ILA no limite superior da normalidade, ainda assim sofreram uma variação importante, para mais, em seu ILA. As variações nos valores deste índice não são fáceis de explicar por este experimento. Existem vários mecanismos que podem atuar na regulação da produção, absorção e nas vias de trocas materno-fetais do LA, embora ainda estejam pouco esclarecidos ${ }^{3}$.

Em nosso estudo, antes do experimento, valores de ILA muito baixos foram identificados em algumas gestantes clinicamente normais, em diferentes idades gestacionais. Todas apresentaram aumentos significativos no ILA após a imersão em água, semelhante ao descrito por Strong Jr. ${ }^{10}$. Sugere-se que isto aconteça em razão do grau de hidratação prévio da gestante e/ou do feto e da taxa de filtração renal fetal prévia, naquele período gestacional.

Um aspecto muito interessante é a relação existente entre o volume intravascular materno e o volume de LA. Goodlin et al. ${ }^{19}$ e Sherer et al. ${ }^{9}$, por exemplo, observaram que mulheres grávidas supostamente hipovolêmicas apresentaram um aumento no volume de LA após a ingestão oral ou endovenosa exagerada de líquidos, revertendo inclusive a oligoidramnia presente.

$O$ repouso no leito e a imersão em água têm sido empregados no tratamento de doenças há muitos séculos. Uma combinação de mudanças na pressão hidrostática, na redução do metabolismo e na compressão do peso sobre o sistema músculo-esquelético, bem como os benefícios psicológicos associados com o repouso, leva à resposta adaptativa $^{7-10,18}$.

Por outro lado, há longo tempo têm sido observados os beneficios da atividade física dentro da água. Em particular, neste trabalho, enfatizamos a pressão hidrostática da água (Lei de Pascal). Resumindo, a água exerceria uma pressão adicional sobre cada ponto da superficie do corpo imerso, aumentando com a densidade da água e a profundidade da banheira. Conforme estudos prévios, se a pessoa estiver em pé na água ou submersa, seu corpo sofre uma pressão maior nos membros inferiores, com alterações no volume dos líquidos intra e extravasculares, auxiliando o retorno venoso com conseqüente maior débito cardíaco ${ }^{17}$. Em conjunto, todas estas propriedades resultam em uma correção postural e do edema da grávida, na redistribuição dos fluidos corporais e em um maior aporte de nutrientes e fluxo ao feto ${ }^{7}$.

Risch et al. ${ }^{17}$, estudando os efeitos imediatos do grau da imersão em água sobre a circula- ção central, em homens, encontraram que o volume cardiaco aumentava de aproximadamente 130 $\mathrm{mL}$ (com a água ao nível do diafragma) para 250 $\mathrm{mL}$ (com a água ao nivel do pescoço), enquanto que a pressão venosa central aumentava de $2,5 \mathrm{mmHg}$ para $12,8 \mathrm{mmHg}$, com queda de cerca de $15 \%$ na freqüência cardíaca.

Greenleaf et al. ${ }^{20}$ observaram, em homens saudáveis, um aumento no volume plasmático de 8,8\% logo após 30 minutos de imersão em água a $34,5^{\circ} \mathrm{C}$. Epstein et al. ${ }^{13}$ estimaram que a expansão do volume intravascular, produzida pela imersão subtotal do corpo humano em água, é equivalente àquela produzida pela infusão endovenosa de 2 litros de uma solução salina isotônica.

Uma explicação para a alteração de volume do LA pode estar na presença de uma provável redistribuição dos fluidos corporais do espaço intersticial para o espaço intravascular, ocasionadas pela pressão hidrostática da água sobre os membros inferiores da mãe, com conseqüente elevação do volume plasmático, aumento do débito cardíaco materno e maior oferta ao compartimento uterino ${ }^{12-17,19,20}$.

Apesar de estarmos conscientes das limitações impostas pelo tamanho dos nossos subgrupos de experimento, este é o primeiro estudo que demonstrou haver modificações no ILA, na PAM, e no pulso, em gestantes, quando submetidas à imersão corporal subtotal em água aquecida, em nosso meio.

Frente a estas considerações e aos resultados obtidos neste trabalho, sugere-se que a técnica de imersão subtotal em água aquecida, para gestantes acima de 28 semanas, pode ser uma medida terapêutica adequada e segura quando se objetiva aumentar o volume de líquido amniótico, na presença de bolsa íntegra, em condições normais e patológicas. O tempo de imersão de $30 \mathrm{mi}-$ nutos é tão eficaz e suficiente quanto 45 e $60 \mathrm{mi}$ nutos. A imersão em água é mais segura, prática e rápida do que as outras técnicas que visam aumentar o VLA e mobilizar os fluidos extravasculares durante a gestação.

\section{SUMMARY}

Purpose: to study the changes in amniotic fluid index (AFI) measured by ultrasonography, mean arterial pressure (MAP) and pulse rate in normal pregnant women submitted to subtotal body immersion in water for different periods of 
time, and to standardize the technique.

Methods: AFI values were studied as recommended by Phelan et al. ${ }^{1}$ in a group of 52 pregnant women with gestational age of 28 weeks or more considered to be clinically normal, before and after exposure to subtotal immersion in water heated to 32 to $34^{\circ} \mathrm{C}$ for 30, 45 and $60 \mathrm{~min}$. The patients were seen at the Ultrasonography and Medical Updating School of Ribeirão Preto and in the Department of Gynecology and Obstetrics of the Faculty of Medicine of Ribeirão Preto, University of São Paulo.

Results: before and after immersion, the average of MAP was 87.8 and 87.1 in the group of $30 \mathrm{~min}, 76.7$ and 66.6 in the group of $45 \mathrm{~min}$ and 77.4 and 60.7 in the group of $60 \mathrm{~min}$, with statistical significance. Before and after immersion, the means of pulse rates were 74.9 and 78.7 in the group of 30 min, 83.6 and 85.2 in the group of 45 min and 84.9 and 90.6 in the group of $60 \mathrm{~min}$, with statistical significance. The mean of AFI also showed statistical significance. When submitted to 30-min immersion the means of AFI were $11.7 \mathrm{~cm}$ before and $16.8 \mathrm{~cm}$ after. In the group of $45 \mathrm{~min}$, the averages were $9.7 \mathrm{~cm}$ before and $13.8 \mathrm{~cm}$ after immersion. In the group of 60 min, the averages were $9.5 \mathrm{~cm}$ before and $13.6 \mathrm{~cm}$ after immersion. The time of immersion of 30-min was as effective and ample as 45 or $60 \mathrm{~min}$.

Conclusions: subtotal immersion in water is a safe and practical procedure that can mobilize fluids during pregnancy, increasing amniotic fluid volume.

KEY WORDS: Amniotic fluid index. Amniotic fluid, subtotal immersion in water. Pregnancy, normal.

\section{Referências}

1. Phelan JP, Smith CV, Broussard P, Small M. Amniotic fluid volume assessment using the fourquadrant technique at 36-42 weeks' gestation. J Reprod Med 1987; 32:540-2.

2. Brace RA. Current topic: progress toward understanding the regulation of amniotic fluid volume: water and solute fluxes in and through the fetal membranes. Placenta 1995; 16:1-18.

3. Gilbert WM, Brace RA. Amniotic fiuid volume and normal flows to and from the amniotic cavity. Semin Perinatol 1993; 17:150-7.

4. Marsden D, Huntingford PJ. An appraisai of the Coomassie blue dilution technique for measuring the volume of liquor amnii in late pregnancy. J Obstet Gynaecol Br Commonw 1965; 72:65-8.

5. Moore TR, Cayle JE. The amniotic fluid index in normal human pregnancy. Am J Obstet Gynecol 1990; 162:1168-73.
6. Novais DA, Cunha SP, Duarte G. Amnioinfusão em rotura prematura de membranas ovulares. Rev Bras Ginecol Obstet 1996; 18:587-94.

7. Katz VL, Ryder RM, Cefalo RC, Carmichael SC, Goolsby R. A comparison of bed rest and immersion for treating the edema of pregnancy. Obstet Gynecol 1990; 75:147-51.

8. Rudge MVC, Berezowski AT, Peraçoli JC, Missiato M. Técnicas para aumentar líquido amniótico. Femina 1993; 21:902-11.

9. Sherer DM, Cullen JB, Thompson HO, Woods JR Jr. Transient oligohydramnios in a severely hypovolemic gravid woman at 35 weeks' gestation with fluid reaccumulating immediately after intravenous maternal hydration. Am J Obstet Gynecol 1990; 162:770-1.

10.Strong TH Jr. Reversal of oligohydramnios with subtotal immersion: a report of five cases. Am J Obstet Gynecol 1993; 169:1595-7.

11.Epstein M, Norsk P, Loutzenhiser R, Sonke R. Detailed characterization of a tank used for headout water immersion in humans. J Appl Physiol 1987; 63:869-71.

12.Doniec-Ulman I, Kokot F, Wambach G, Drab M. Water immersion-induced endocrine alterations in women with EPH gestosis. Clin Nephrol 1987; 28:51-5.

13.Epstein M. Water immersion and the kidney: implications for volume regulation. Undersea Biomed Res 1984; 11:113-21.

14.Goodlin RC, Hoffman KL, Williams NE, Buchan P. Shoulder-out immersion in pregnant women. J Perinat Med 1984; 12:173-7.

15.Greenleaf JE. Physiological responses to prolonged bed rest and fluid immersion in humans. J Appl Physiol 1984; 57:619-33.

16. Khosla SS, DuBois AB. Osmoregulation and interstitial fluid pressure changes in humans during water immersion. J Appl Physiol 1981; 51:686-92.

17.Risch WD, Koubenec HJ, Beckmann U, Lange S, Gauer $\mathrm{OH}$. The effect of graded immersion on heart volume, central venous pressure, pulmonary blood distribution, and heart rate in man. Pflügers Arch 1978; 374:115-8.

18.Katz VL, McMurray R, Berry MJ, Cefalo RC. Fetal and uterine responses to immersion and exercise. Obstet Gynecol 1988; 72:225-30.

19.Goodlin RC, Anderson JC, Gallagher TF. Relationship between amniotic fluid volume and maternal plasma volume expansion. Am J Obstet Gynecol 1983; 146:505-11.

20.Greenleaf JE, Morse JT, Bames PR, Silver J, Keil LC. Hypervolemia and plasma vasopressin response during water immersion in men. J Appl Physiol 1983; 55:1688-93. 